Mittheilungen aus dem chemischen Institut der Universität Göttingen.

\title{
Zur Theorie der Oxydationsprocesse;
}

von W. Manchot.

(Eingelaufen am 21. September 1902.)

Wenn man die Literatur über die sogenannte, Sauerstoffactivirung" durchsieht, so fällt auf, dass wohl auf keinem Gebiete der Chemie die Publicationen experimentellen Inhalts an Zahl und Umfang von den theoretischen Betrachtungen so sehr übertroffen werden, wie hier. Verdienstvoller als die letzteren zu vermehren, erschien es mir deshalb, sicher begrundetes experimentelles Beobachtungsmaterial herbeizuschaffen, $d$. h. neue Thatsachen aufzufinden, welche für sich allein - ganz unabhängig von der theoretischen Erklärung die man bevorzugt - ihre Bedeutung haben. In meinen früheren Mittheilungen über diesen Gegenstand habe ich deshalb absichtlich einer Discussion der älteren Theorien wenig Raum gegeben und andererseits die allgemeinen Schlussfolgerungen, welche ich aus meinen Versuchen zog, meist nur angedeutet.

Gegenüber der zunehmenden Bearbeitung des Gebietes ist dieses Verfabren jedoch nicht mehr durchfuhrbar und so wolle man mir gestatten, meine Ansichten thber die Vorgänge, um die es sich hier handelt, nunmehr auseinanderzusetzen.

Von allen Theorien die man aufgestellt hat, um die 0xydationswirkung des freien Sanerstoffs zu erklären, scheint gegenwärtig nur eine im Stande $z u$ sein den Thatsachen in genügender Weise gerecht zu werden, nämlich die Superoxydtheorie, welche $1897 \mathrm{Bach}^{1}$ ) und Engler ${ }^{2}$ ) unabbängig von einander aufgestellt und experimentell zu begründen sich bemüht

1) Bach, Compt. rend. 124, 951; Chem.-Zeitg. 1897, 398, 436.

2) Engler und Wild, Ber. d. deutsch. chem. Ges. 30, 1669. 
haben, ersterer durch qualitative Versuche, letzterer durch eingehende quantitative und qualitative neue Beobachtungen. Engler und Wöhler ${ }^{3}$ ) haben gegen Ende vorigen Jahres diese Theorie in einleuchtender Weise nochmals dargelegt und dabei den Versuch gemacht, sie auch auf andere Oxydationsprocesse - so die Wirkung des Wasserstoffsuperoxyds - zu übertragen. Etwas vorher habe ich eine experimentelle Abhandlung ${ }^{4}$ ) publicirt, welche von dem gleichen Gedanken veranlasst war, $d . h$. von dem Gedanken, dass wenn thatsächlich Superoxyde unter der Einwirkung von Sauerstoffgas entstehen, solche auch unter der Einwirkung anderer Oxydationsmittel entstehen müssen. Dieser Gedanke wurde für den Fall Eisenoxydul + Wasserstoffsuperoxyd als richtig nachgewiesen. Ich habe nun zusammen mit C. Wilhelms weiter in dieser Richtung gearbeitet und dabei hat sich ergeben, dass allgemein durch die Einwirkung ron Oxydationsmitteln auf Ferroverbindungen primär ein Peroxyd ${ }^{5}$ ) entsteht. Es liegt sehr nahe, die Ueberlegung, von der aus diese Resultate gewonnen wurden, zu verallgemeinern. Dann ergeben sich zwei wichtige Schlussfolgerungen :

1) Da die Einwirkung der verschiedensten Oxydationsmittel auf eine oxydable Substanz von Erscheinungen begleitet ist, welche den bei der Luftoxydation beabachteten qualitativ sehr nahe stehen und quantitativ ein Unterschied nur hinsichtlich der Anzahl der verbrauchten Aequivalente Sauerstoff beobachtet wird, so sind alle Theorien hinfällig, welche eine specielle Erklärung der Autoxydation involviren, d. h. auf andere Oxydationsprocesse nicht übertragbar sind. Selbstverständlich folgt hieraus weiter, dass es nicht nothwendig ist, die Activirung des freien Sauerstoffs (Peroxydbildung) stets der Anlagerung von ganzen

\footnotetext{
3) Zeitschr. f. anorg. Chem. 29, 1 (4. December 1901).

4) Manchot und Wilhelms, Ber. d. deutsch. chem. Ges. 34, 2479 (28. September 1901).

s) Vergl. die folgende Abhandlung.
} 
Molekülen Sauerstoff zuzuschreiben, wie dies Bach und Engler bisher gethan haben.

2) gelange ich - ohne Zweifel in der Hauptsache in völliger Uebereinstimmung mit Engler - zu der folgenden allgemeinen Theorie der Oxydationsprocesse, welche vollständig enthalten ist in dem Satze: "Bei allen Oxydationsprocessen entsteht ein „Primäroxyd“, welches im Allgemeinen den Charakter eines Peroxyds besitzt" "6).

Das weitere Schicksal dieses Primäroxyds wird im Einzelfalle durch die speciellen Verhältnisse bedingt. Der Fall, dass das fassbare, resultirende Product mit dem Primäroxyd identisch ist, scheint der seltenere zu sein. Er scheint aber bei organischen Substanzen in der letzten Zeit insbesondere von Baeyer und Villiger ${ }^{7}$ ), Engler und Frankenstein ${ }^{8}$ ), Bamberger ${ }^{9}$ ) und Wolffenstein ${ }^{10}$ ) wiederholt beobachtet worden zu sein.

Ferner ist die Verbrennung von Wasserstoff zu nennen, welche primär Wasserstoffsuperoxyd - nicht Wasser - orgiebt, sowohl in der Flamme (Traube), wie an der Kathode (Traube $\left.{ }^{11}\right)$, Richarz $\left.{ }^{12}\right)$ ). An diese schliesst sich an die von Herzog und mir ${ }^{13}$ ) studirte Autoxydation des Kobaltcyanurs, bei welcher der nascente Wasserstoff durch Sauerstoffgas in

8) Eine scharfe Unterscheidung zwischen ,activirtem" oder "Peroxyd"Sauerstoff einerseits und dem zur Bildung des "gewöhnlichen" Oxydationsproductes erforderlichen Sauerstoff andererseits ist dem Sinne der Theorie nach weder möglich noch nöthig. Deshalb schlage ich hier die Bezeichnung "Primäroxyd" vor.

7) Ber. d. deutsch. chem. Ges. $\mathbf{3 8}$ und $\mathbf{3 4}$.

8) Ber. d. deutsch. chem. Ges. 34, 2933.

9) Ber. d. deutsch. chem. Ges. 32, 1882; 34, 12.

10) Ber. d. deutsch. chem. Ges. 34, 2933.

11) Traube, Ber. d. deutsch. chem. Ges. 26, 1473; vergl. Engler, ebenda $\mathbf{3 3}, 1103$.

19) Richarz, Zeitschr. f. physik. Chem. 20, 145 .

18) Manchot und Herzog, Ber. d. deutsch. chem. Ges. 33, 1746; Zeitschr. f. anorg. Chem. 27, 431. 
$\mathrm{H}_{2} \mathrm{O}_{2}$ umgewandelt wird, während zugleich durch eine concurrirende aber viel langsamere Reaction ein Bruchtheil des Cyanürs direct peroxydirt wird. Analog denke ich mir die Autoxydation von Metallen wie Zink und Blei verlaufend.

Von anorganischen Substanzen ist weiter das durch Luftoxydation entstehende Peroxyd der Stufe $\mathrm{CeO}_{3}{ }^{14}$ ) zu nennen.

Häufiger ist namentlich bei anorganischen Verbindungen der Fall, dass das primäre Oxydationsproduct sich zersetzt, ehe man seiner hablaft werden kann, indem es Sauerstoff abgiebt und zwar entweder direct als Gas oder an andere Substanzen ("Acceptoren" nach Engler), welche zugegen sind. Ein solcher Acceptor kann auch die Substanz selbst, welche oxydirt wird, mit ihrem noch unoxydirten Theile ${ }^{15}$ ) sein (,Selbstacceptoren"). Die Aufdeckung der Reactionen letzterer Art, der secundären Reactionen, ist wesentlich für die Erkenntniş der Vorgänge und hierbei wiederum $\mathrm{zu}$ beachten, dass die secundären Reactionen andere, oft grössere, Geschwindigkeit haben können als die primäre. Hierauf brauche ich nicht weiter einzugehen, da diese Verbältnisse in Engler's und meinen Arbeiten genügend dargelegt sind.

Dagegen möchte ich die Bildung eines Primäroxyds und seinen Zerfall zum resultirenden Producte noch etwas näher auseinandersetzen. Hierbei verdient nämlich eine Erscheinung ganz besonders hervorgehoben zu werden. Das primäre Peroxyd bildet sich direct aus der reagirenden Oxydationsstufe. Die Zwischenstufen werden übersprungen. Die von meinen Mitarbeitern und mir angestellten Versuche über die Oxydation der Ferroverbindungen durch $\mathrm{O}_{2}{ }^{16}$ ), durch $\mathrm{H}_{2} \mathrm{O}_{2}{ }^{17}$ ) und nament-

14) Job, Ann. de chim. [7] 20, 264.

15) D. h. mit den Theilchen (Ionen, Molekülen, Complexen), welche mit dem Oxydans noch nicht in Berührung gekommen sind. Bei organischen Substanzen wirkt wohl auch der Rest des angegriffenen Moleküls als Acceptor.

18) Zeitschr. f. anorg. Chem. 27, 430 .

$\left.{ }^{17}\right)$ Ber. d. deutsch. chem. Ges. 34, 2479. 
lich durch Chromsäure ${ }^{18}$ ) zeigen dies auf das Schlagendste ${ }^{19}$ ). Ferner hat $-\mathrm{J}_{0} \mathrm{~b}^{20}$ ) bei der Luftoxydation von Cerocarbonat die Bildung eines Salzes des sechswerthigen Cers beobachtet. . Dieses

Salz entstand nicht, wenn man ron Ce-Salz ausging; also bildet sich die Stufe $\stackrel{\text { Ve }}{ } \operatorname{direct}^{21}$ ) aus $\mathrm{II}$

Sind also $\stackrel{\text { II }}{M}$ bis $\mathrm{VI}$ die Oxydationsstufen eines Metalls nach seiner Werthigkeit, so hat man z. B. für Eisenoxydul + Sauerstoffgas:

$$
\stackrel{\mathrm{II}}{\mathrm{M}} \rightarrow \stackrel{\mathrm{IV}}{\mathrm{M}} \rightarrow \stackrel{\mathrm{III}}{\mathrm{M}}
$$

und für Eisenoxydul + Chromsäure (oder + Wasserstoffsuperoxyd):

$$
\stackrel{\mathrm{II}}{\mathrm{M}} \rightarrow \stackrel{\mathrm{V}}{\mathrm{M}} \rightarrow \stackrel{\mathrm{III}}{\mathrm{M}}
$$

Nun ist es offenbar nur ein Specialfall, dass wie beim Eisen die resultirende fassbare Oxydationsstufe höher liegt, als die Anfangsstufe, denn es sind mehrere verschiedene Möglichkeiten denkbar: Die Endstufe kann mit der Anfangsstufe identisch

18) Vergl. die folgende Abhandlung.

19) Die experimentelle Eriahrung entspricht also hier durchaus der von Ostwald (Zeitschr. f. phys. Chem. 22, 306; 34, 252) aufgestellten Regel, nach der sich bei einem Processe primär nicht die stabilsten Producte bilden, sondern die am leichtesten erreichbaren, also gerade labile Producte.

so) loc. cit.

21) Durch Einwirkung von $\mathrm{CeO}_{3}$ auf Cerosalz bildet sich hierbei ein wenig Cerisalz. Mit Hülfe der Methode die ich früher beim Eisen angewendet habe, gelang es jedoch kürzlich Baur (Zeitschr. f. anorg. Chem. 30, 251! den gesammten Sauerstoff, der dem Uebergange von $\mathrm{Ce}_{2} \mathrm{O}_{3}$ in $2 \mathrm{CeO}_{2}$ über $2 \mathrm{CeO}_{3}$ entspricht, zur Absorption zu bringen. Damit ist bewiesen, dass jenes $\mathrm{CeO}_{3}-\mathrm{Salz}$ ein Primäroxyd ist.

Die Absorption bricht offenbar nicht ab, wenn der Acceptor auch die Ceri-Stufe reducirt, wie Job (Compt. rend. de l'Acad. de sc. Mai 1902) sehr schön demonstrirt. Analoge Verhältnisse habe ich bereits beim Eisen beschrieben (Zeitschr. f. anorg. Chem. 27, 430). 
sein (Beispiel : Ferrioxyd + Wasserstoffsuperoxyd ${ }^{92}$ )) oder unter ihr liegen, d. h. der Zerfall des Primäroxyds führt zu derjenigen Stufe, die unter den gegebenen Bedingungen stabil ist, und die Zwischenstufen werden übersprungen oder sehr rasch durchlaufen, weil sie unter den Versuchsbedingungen nicht existenzfähig sind.

So erklärt sich die "Reduction" von $\mathrm{PbO}_{2}$ oder $\mathrm{MnO}_{2}$ durch $\mathrm{H}_{2} \mathrm{O}_{2}$ bei Gegenwart von Säure

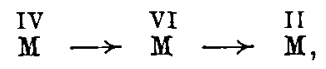

entsprechend den von Lunge ${ }^{23}$ ) angegebenen quantitativen Verhältnissen.

Während ich diese Auffassung als allgemein für die Wirkung der Oxydationsmittel gültig mir aus meinen Versuchen am Eisen seit einiger Zeit abgeleitet habe, hat vor kurzem $\mathrm{Bach}$ nur für den Specialfall der Wirkung von $\mathrm{H}_{9} \mathrm{O}_{2}$ eine ähnliche Ansicht entwickelt, um dessen katalytische Zersetzung durch Peroxyde wie $\mathrm{PbO}_{2}$ u. a. $\mathrm{zu}$ erklären und hat dieselbe durch Versuche über die Einwirkung von Chromsäure auf Wasserstoffsuperoxyd zu begründen sich bemüt ${ }^{24}$ ).

Meine Anschaungen sind demnach gegenuber denjenigen von Bach noch allgemeinerer Natur und gehen uber dieselben hinaus.

Der Erwähnung werth ist noch, dass das aus dem Zerfall resultirende Product das Metall selbst sein kann. Beispiele sind die "Reduction“ von Goldchlorid durch alkalisches Wasserstoffsuperoxyd, ferner die ,katalytische" Zersetzung von Wasserstoffsuperoxyd durch Platin.- Meine Auffassung dieser Katalyse

22) Manchot und Wilhelms, Ber. d. deutsch. chem. Ges. 34, 2486.

$\left.{ }^{23}\right)$ Ber. d. deutsch. chem. Ges. 18, 1872. Chem.-techn. Untersuchungsmeth. Berlin 1899, II, 506.

24) B a ch, Ber. d. deutsch. chem. Ges. 35, 876. Dass die quantitativen Verhältnisse hierbei nicht einer glatten „Reduction" der Chromsäure entsprechen, zeigen auch eine Reihe älterer Angaben z. B. Asch off, Journ. f. pract. Chem. 81, 407 (1860). 
unterscheidet sich also von der Traube'schen ${ }^{25}$ ), die Bredig ${ }^{26}$ ) vertritt, und der auch Engler und Wöhler sich zunächst noch anzuschliessen scheinen. Nach Bredig beruht die Katalyse des Wasserstoffsuperoxyds durch Platin auf einer Oxydation zu einem Platinoxyd, welches dann durch weitere Einwirkung von $\mathrm{H}_{2} \mathrm{O}_{2}$ reducirt wird, wäbrend nach dem Obigen nur Oxydation des Platins und Zerfall des Platinperoxyds anzunehmen wäre.

Hiergegen wird man einwenden, dass die Einwirkung von $\mathrm{H}_{2} \mathrm{O}_{2}$ auf $\mathrm{PbO}_{2}$ u. a. durch das bekannte "Reductionsvermögen“" des Hydroperoxyds zu erklären sei; dem gegenüber behaupte ich: das Wasserstoffsuperoxyd ist kein Reductionsmittel sondern nur Oxydationsmittel ${ }^{27}$ ). Mag man nun diese Ansicht auch zunächst eine rein subjective nennen, so lässt sich doch leicht zeigen, dass sie der bisherigen von der chemischen Doppelnatur des Wasserstoffsuperoxyds mindestens gleichberechtigt gegenuber steht; denn diese ist sehr anfechthar.

Betrachten wir zunächst die von Weltzien herrührende und von Traube bevorgugte Annahme, für die sich auch Baey e ${ }^{28}$ ) - allerdings ehe er die Vierwerthigkeit des Sauerstoffs anerkannt hatte - ausgesprochen bat, nach welcher $\mathrm{H}_{\mathbf{z}} \mathrm{O}_{\mathbf{g}}$ ein mit zwei Wasserstoffatomen reagirendes R $\epsilon$ ductionsmittel sein soll. Thatsächlich sind Reactionen, die in diesem Sinne gedeutet werden müssen, bisher noch nicht bekannt geworden. So ist bei den zahlreichen organischen Substanzen, welche namentlich von Ba eyer und Villiger, ferner von Wolffenstein und von $\mathrm{Bamberger}$ mit $\mathrm{H}_{2} \mathrm{O}_{2}$ bebandelt worden sind, noch niemals eine Anlagerung von Wasserstoff beobachtet worden, wie sie von einem mit zwei Wasserstoffatomen reagirenden Reductionsmittel zu erwarten wäre, sondern stets ist nur eine

95) Ber. d. deutsch. chem. Ges. 15, 672; vergl. Engler und Wöhler, Zeitschr. f. anorg. Chem. $\mathbf{2 9}, 1$.

s6) Zeitschr. f. physik. Chem. 37, 5.

27) Wie jedes schwächere Oxydans im Vergleich zu einem stärkeren kann man es natürlich ein Reductionsmittel nennen.

28) Ber. d. deutsch. chem. Ges. 33, 2495. 
Addition von Sauerstoff eingetreten. Ferner lassen sich alle Reactionen von $\mathrm{H}_{8} \mathrm{O}_{3}$, in denen man eine Bethätigung der beiden Wasserstoffatome erblickt hat, ebenso ungezwungen oder vielmehr besser auf das Verbalten des activen Sauerstoffatoms in $\mathrm{H}_{2} \mathrm{O}_{2}$ zuruckfuhren. Vorländer ${ }^{29}$ ) macht zu Gunsten jener vermeintlichen Wasserstoffwirkung geltend, dass Aethylbydroperoxyd und noch mehr Diätbylhydroperoxyd gegen Uebermangansäure viel beständiger ist, als Hydroperoxyd. Diese Argumentation ist jedoch durchaus nicht stichhaltig, denn Diätbylhydroperoxyd ist nach der Beschreibung seines Entdeckers ${ }^{30}$ ) nicht nur gegen $\mathrm{HMnO}_{4}$ sondern auch gegen Reductionsmittel viel beständiger als $\mathrm{H}_{3} \mathrm{O}_{2}$. Daraus folgt, dass eben das Sauerstoffatom, welches sich bei den Reactionen von $\mathrm{H}_{2} \mathrm{O}_{2}$ bethätigt, hier viel fester gebunden, ist als im Hydroperoxyd. Es liegt nahe, bezüglich der Ursache dieser Erscheinung an eine ăhnliche Wirkung der Substitution auf die dritte und vierte Valenz des Sauerstoffatoms zu denken, wie sie für die vierte und funfte Valenz des Stickstoffatoms bekannt ist:<smiles>[Z1]O[O]</smiles><smiles></smiles>

Ferner ist wohl verständlich, dass $\mathrm{H}_{2} \mathrm{O}_{2}$ auf die niederen Oxyde des Mangans anders einwirkt als auf die des Eisens, nämlich bei dem einen Metall eine höhere Oxydationsstufe erzeugt als bei dem anderen $\left(\mathrm{MnO}_{3}\right.$ bezw. $\left.\mathrm{Fe}_{9} \mathrm{O}_{6}\right)$, aber nicht, dass diese Einwirkung eine principiell verschiedene, nämlich beim Mangan eine reducirende, boim Eisen eine oxydirende sei.

In der That lassen sich alle Reactionen des $\mathrm{H}_{2} \mathrm{O}_{2}$ mindestens ebenso ungezwungen auf seine oxydirende wie auf die reducirende Wirkung zurückführen. $\mathrm{Für}^{\mathrm{PbO}} \mathrm{PbnO}_{2}$ ist dies oben schon gezeigt. Auch bei NaOCl liegt keine Schwierigkeit vor. Die Einwirkung auf Ferricyankalium ist keine glatte

$\left.{ }^{29}\right)$ Dieso Annalen 320, 311.

30) B aөyer, Ber. d. deutsch. chem. Ges. 33, 3390 . 
„Reduction“ sondern zeigt eigenthümliche Unregelmässigkeiten $^{31}$ ).

Bezüglich Uebermangansäure und $\mathrm{H}_{2} \mathrm{O}_{2}$ besteht ein Streit 2wischen Baeyer und Bach, welcher Letztere die Entstehung von $\mathrm{H}_{2} \mathrm{O}_{4}$ behauptet. Dieser Streit ist für die Frage, die ich hier erörtere, insofern ohne Belang, als $\mathrm{H}_{2} \mathrm{O}_{2}$ und $\mathrm{HMnO}_{4}$ wohl im Verbältniss von zwei verschieden starken Oxydationsmitteln $\mathrm{zu}$ einander stehen mögen. Eine Oxydation von $\mathrm{HMnO}_{4}$ durch $\mathrm{H}_{2} \mathrm{O}_{2}$ ist andererseits auch nicht gerade wahrscheinlich. Fur die Umsetzung in verdünnter Lösung kommt aber noch eine dritte Erklärung in Betracht. Bekanntlich wirkt reines $\mathrm{H}_{8} \mathrm{O}_{2}$ auf frisch bereitetes Permanganat gar nicht sofort ein. Die Entfärbung erfolgt erst nach einiger Zeit. Die Reaction geht aber flott weiter, wenn sie einmal angefangen hat und die Verzögerung bleibt aus, wenn man eine alte Permanganatlösung nimmt oder von vornherein Mangansulfat zusetzt. Der Fall liegt also ähnlich wie bei der Titration der Oxalsäure. $\mathrm{H}_{2} \mathrm{O}_{2}$ reagirt hier nicht mit $\mathrm{HMnO}_{4}$ selbst, sondern mit dem Manganperoxyd, welches durch Einwirkung von $\mathrm{HMnO}_{4}$ auf $\mathrm{MnSO}_{4}$ entsteht. Diese Erklärung stützt sich also auf die bekannten Manganstudien von Volhard ${ }^{32}$ ) und Zimmermann ${ }^{39}$ ).

Die traditionelle Auffassung, dass $\mathrm{H}_{2} \mathrm{O}_{2}$ zugleich Oxydationsund Reductionsmittel sei, scheint mir demnach unhaltbar $z u$ sein. Näher kommt der hier vertretenen Auffassung die Ansicht Schönbein's, nach welcher der freiwerdende Sauerstoff zur Hälfte aus $\mathrm{H}_{2} \mathrm{O}_{2}$, zur Hälfte aus $\mathrm{PbO}_{2}$ u. s. w. stammen soll, insofern diese Auffassung ja eine wenn auch noch so kurze Anlagerung von $\mathrm{H}_{2} \mathrm{O}_{2}$ an das betreffende Peroxyd voraussetzt. Jedoch ist gegen sie das principielle Bedenken zu erheben, dass sie, ebenso wie die Ansicht von Weltzien-Traube, quantitative Umsetzungen a priori festlegt, welche nur auf ex-

s1) Vergl. Manch ot, Ber. d. deutsch. chem. Ges. 33, 1743 und die folgende Abhandlung.

39) Diese Annalen 198, 318 .

38) Diese Annalen $\mathbf{2 1 3}, 305$. 
perimentellem Wege von Fall zu Fall ermittelt werden können. Wenn nämlich auch die Schönbein'sche Auslegung und die hier vertretene Superoxydtheorie für Specialfälle wie $\mathrm{PbO}_{2}$ u. s. w. zu derselben Umsatzgleichung führen, so ist diese Uebereinstimmung docb nur eine zufällige. Wird nämlich z. B. ein Oxyd $\mathrm{MO}_{2}$ zu einem höheren oxydirt, das dann $\mathrm{zu}$ dem resultirenden MO zerfällt, so hat man nach der Superoxydtheorie $\mathrm{zu}$ formuliren:

$$
\mathrm{MO}_{2}+\mathrm{xH}_{2} \mathrm{O}_{2}=\mathrm{MO}_{2}+\mathrm{x}+\mathrm{xH}_{2} \mathrm{O}=\mathrm{MO}+\mathrm{O}_{1+x},
$$

woraus z. B. für $x=1 / 2$ folgt, dass $1 \frac{1 / 2}{0}$ frei werden, während nach Weltzien-Traube und Schönbein a priori die Gleichungen

$$
\mathrm{MO}_{2}+\mathrm{H}_{2} \mathrm{O}_{2}=\mathrm{MO}+\mathrm{H}_{2} \mathrm{O}+2 \mathrm{O}
$$

nöthig sind. In der That stimmt, wie oben erwăhnt, die Umsetzung von $\mathrm{CrO}_{3}$ mit $\mathrm{H}_{2} \mathrm{O}_{2}$ mit der nach $\mathrm{Schönbein} \mathrm{formu-}$ lirten Gleichung nicht überein.

Man wird nun einwenden, dass diesen Anschauungen zufolge für viele Elemente Oxydationsstufen angenommen werden müssen, die man noch nicht kennt. In der That möchte ich eben diesen Schluss ziehen. Die Fähigkeit der Elemente Superoxyde zu bilden ist nach, den Erfahrungen der letzten Jahre ohne Zweifel viel grösser als die bisher isolirten Oxyde anzeigen, und eine experimentelle Durchforschung des ganzen Systems nach dieser Richtung balte ich für eine wichtige Aūfgabe der anorganischen Chemie. Ihre Durchfuhrung, die freilich Hülfsmittel für andauerndes Operiren bei extremen Temperaturen erfordert, würde sicher auch unsere Kenntniss vom Wesen der Valenz erheblich fördern. Denn offenbar sind die Superoxyde noch nicht die primären Producte der Einwirkung eines Oxydationsmittels auf eine Substanz: der Bildung dieser Oxydationsstufen muss vielmehr eine Anlagerung der reagirenden Theilchen an einander vorausgehen. Diese Anlagerungsproducte werden sich, wenn das Oxydans $\mathrm{O}_{2}$ oder $\mathrm{H}_{2} \mathrm{O}_{2}$ ist, oft von höheren "Oxyden" kaum unterscheiden lassen. Als hierher 
gehörig betrachte ich z. B. die Oxydation von Indigweiss, Oxanthranol ${ }^{34}$ ) u. a. Bei anderen Oxydantien aber wird ein solcher Unterschied schärfer hervortreten. Die Hoffunng, diese primären Anlagerungsproducte isoliren zu können, ist im Allgemeinen nicht gross. Dennoch wird man sie, wie jedes Zusammentreffen von Separattheilchen ${ }^{35}$ ), bei welchem eine Zustandsänderung dieser Theilchen (Austausch elektrischer Ladung, calorischer Effect, Entstehung neuer Theilchen) eintritt, als chemische Verbindungen ansehen und danach trachten müssen ibre Entstehung nachzuweisen, mag ihre Existenzdauer ${ }^{36}$ ) auch noch so kurz sein. Dabei wird man freilich mit dem Valenzschema, $d$. h. mit der Vorstellung, dass es sich bei der Bildung von chemischen Verbindungen stets um eine Absättigung von Valenz durch Valenz handelt, nicht mehr durchkommen, sondern wird Vorstellungen heranzieben müssen wie sie Werner entwickelt hat, nach denen eine Anlagerung auch durch eine von der Gesammtheit des Complexes ausgehende Attraction bewirkt werden kann.

Die letaten Betrachtungen kann man offenbar auch in umgekehrter Reihenfolge anstellen, also die obige Superoxydtheorie direct ableiten aus den Vorstellungen, welche sich von selbst ergeben, wenn man darüber nachdenkt, wie eine Reaction zu Stande kommt. Solche Vorstellungen hat Kekulé ${ }^{87}$ ) bereits geäussert und seit Jahren findet ja auf organischem Gebiete die Ansicht, dass die Reactionen stets in einer primären Anlagerung bestehen, in $\mathrm{Nef}$ sowie in $\mathrm{Michael}$ eifrige Vertheidiger. Namentlich aber weisen die experimentellen Arbeiten von Thiele über die doppelte Bindung und Untersuchungen

34) Manchot, diese Annalen 314, 177; Manchot und Herzog, ebenda 316, 318.

3s) Moleküle, Atome, Ionen, Complexe.

$\left.{ }^{38}\right)$ Offenbar wäre es ganz willkürlich, wenn man frst von einer bestimmten Existenzdauer an den Begriff der chemischen Verbindung gelten lassen wollte.

37) Lehrbuch I, 142; vergl. MichaeI, Ber. d. dentsch. chem. Ges. 34, 4028. 
104 Manchot, Zur Theorie der Oxydationsprocesse.

von Claisen, Hantzsch, Vorländer und vielen Anderen aber die Wirkung ,negativer" Gruppen nach der von Hen$\mathrm{rich}^{38}$ ) vertretenen Auffassung immer deutlicher auf die Nothwendigkeit hin, die primären Anlagerungsvorgänge zu studiren, wenn man einen wirklichen Einblick in den Mechanismus einer Reaction gewinnen will.

Die vorstehende Theorie der Oxydationsprocesse, welche die Wirkung aller Oxydationsmittel unter einen gemeinsamen Gesichtspunkt bringt, steht somit auch im besten Einklang mit der Entwickelung, welche ein wichtiger Theil der organischen Chemie gegenwärtig nimmt.

Doch möchte ich hervorheben, dass ich ihren Hauptwerth nur darin erblicke, dass sie zu experimenteller Forschung anregt und den Resultaten derselben nicht vorgreift, sondern sich ihnen anzupassen vermag. Sollte letzteres einmal nachweislich nicht mehr zutreffen, so wird man diese Theorie eben bei Seite legen müssen.

88) Vergl. Ber. d. deutsch. chem. Ges. 35, 1773. 